\title{
Association of psoriasis and psoriatic arthritis with systemic lupus erythematosus
}

\author{
Eduardo Bonilla, Ashwini Shadakshari and Andras Perl* \\ Division of Rheumatology, Department of Medicine, SUNY Upstate Medical University, Syracuse, NY 13210, USA
}

\begin{abstract}
Objective: The prevalence of psoriasis (Ps) and psoriatic arthritis (PsA) in patients with systemic lupus erythematosus (SLE) is largely unknown, which was investigated in this study.
\end{abstract}

Methods: The prevalence of Ps and PsA was examined in 445 patients with SLE. The diagnosis of psoriasis was made clinically by a dermatologist or rheumatologist, while the diagnosis of PsA was made on the basis of CASPAR criteria. The diagnosis of SLE was based on the American College of Rheumatology (ACR) classification criteria. Clinical and laboratory variables were compared in SLE patients with and without Ps (SLE/Ps+ and Ps-) as well as in those with and without PsA (SLE/PsA+ and PsA-). Statistical analyses were performed with chi-square test using Graphpad Prism software.

Results: Among 445 patients with SLE, 23 (5.1\%) had Ps, out of which 20 (4.5\%) had PsA which is greater than what is observed in the general population (p < 0.0001). The prevalence of malar rash, discoid rash, photosensitivity, and arthritis were increased in SLE patients with Ps and PsA. Antiphospholipid antibodies (APLAs) were less common in SLE patient with concurrent Ps and PsA.

Conclusions: The prevalence of PsA is increased and it may represent a distinct clinical entity in patients with SLE.

\section{Introduction}

Systemic Lupus Erythematosus (SLE) is an autoimmune systemic inflammatory disease of multifactorial etiology affecting various organs in the body [1]. The etiologic factors involved in the pathogenesis of SLE are not clearly understood and include environmental, hormonal and genetic mechanisms. These trigger the immune system in predisposed individuals leading to inflammation in multiple organs causing their dysfunction. SLE can also be seen as part of an overlap syndrome with other connective tissue disorders such as rheumatoid arthritis, systemic sclerosis, polymyositis, dermatomyositis and Sjogren's syndrome. Occurrence of SLE with concomitant Psoriasis (Ps) has been described [2], but is generally regarded as uncommon. The association of SLE and PsA is also uncommon and minimal data is available regarding their coexistence. Both diseases have a varied clinical presentation which makes the diagnosis difficult at times. The presentation of subacute cutanenous lupus erythematosus (SCLE) may be similar to that of Ps which may lead to confusion the time of diagnosis [3].

Recently, with the increased use of tumor necrosis alpha inhibitors (anti-TNF) for PsA, multiple cases of a lupus like syndrome have been described with some studies showing that it may happen in $0.5-1 \%$ of patients using anti-TNF.

Psoriasis is a common chronic skin disorder affecting more than $2 \%$ of population with a multifactorial cause including genetic, immunologic and environmental factors. It typically presents with erythematous plaques with adherent silver scales but, as SLE, has a variety of clinical forms [4]. Pathogenesis of Ps involves an immunemediated interaction of T-lymphocytes and dendritic cells leading to hyperproliferation of epidermal cells. IL-23 and IL-12 produced by activated myeloid dendritic cells promote the development of Th17 and Th1 cells, respectively. Th17 cells appear to play a major role in the pathogenesis of Ps [5].

Psoriasis may be associated with PsA arthritis in about 30\% of the cases [6]. It may present in a variety of clinical patterns including distal arthritis, asymmetric oligoarthritis, symmetric polyarthritis, arthritis mutilans and as a spondyloarthropathy. Nail involvement and enthesitis may also be seen.

The literature on concomitant occurrence of SLE and Ps is limited to retrospective studies. Case series have described the association between SLE and PsA. In the largest reported retrospective study by Zalla and Muller, 42 out of 9420 Ps patients had SLE giving a prevalence rate of $0.69 \%$ [2]. Janjunratsang et al examined the existence of autoimmunity in Ps and found a prevalence of ANA positivity of less than $2 \%$ in 300 patients with Ps. None of them had been treated with anti-TNF [7].

So far in English-based literature only two patients with concomitant psoriatic arthritis and SLE have been described [8]. In view of this, we decided to do a retrospective study to evaluate the prevalence, clinical and laboratory features of patients with both PsA and SLE.

Correspondence to: Andras Perl, Division of Rheumatology, Department of Medicine, Suny Upstate Medical University, 750 East Adams Street, 8310 Weiskotten Hall, Syracuse, 13210, USA, Tel: 315 464-4194, E-mail: perla@upstate.edu

Key words: SLE, psoriasis, psoriatic arthritis

Received: November 05, 2016; Accepted: December 09, 2016; Published: December 12, 2016 


\section{Methods}

\section{Study population}

A retrospective chart review of all patients with SLE visiting a tertiary, academic rheumatology specialty clinic between 01/01/1990 and $07 / 31 / 12$ was performed. Demographics, medical history, biochemical parameters and radiological findings of patients between the ages of 16 and 85 years were collected. SLE diagnostic features including malar rash, discoid rash, photosensitivity, presence of antiphospholipid antibodies (APLA), seizures, psychosis, oral ulcers, serositis, proteinuria, anemia, leucopenia, thrombocytopenia, hemolytic anemia, anti-Sm, or anti-DNA were also collected from the medical records. Patients were defined as having SLE if they fulfilled four of the 1997 American College of Rheumatology revised criteria [9]. The diagnosis of Ps was established clinically by either a rheumatologist or dermatologist. The diagnosis of PsA was established using the CASPAR (Classification of Psoriatic Arthritis) criteria [10]. Our institutional review board (IRB) approved the protocol and waived the need for an informed consent.

\section{Statistical methods}

The clinical and biochemical features of SLE patients with and without Ps (SLE/Ps+ and Ps-) and with and without PsA (SLE/PsA+ and PsA-) were analyzed with chi-square test using Graphpad Prism software. Statistical difference of $\mathrm{p}<0.05$ was considered significant.

\section{Results}

Between 01/01/1990 and 07/31/12, 445 patients with SLE were identified. Out of these 23 (5.1\%) had Ps and 20 (4.5\% of total patients) had Ps. 20 out of 23 SLE/Ps+ patients had PsA (87\%). There was an increased prevalence of malar rash, discoid rash, photosensitivity, and arthritis in SLE/Ps+ and SLE/PsA+ patients when compared to SLE/ Ps- and SLE/PsA- patients. This was found to be to be statistically significant (Table 1). The presence of APLA was less common in SLE/ Ps+ and SLE/PsA+ patients. There was no significant association between of seizures, psychosis, oral ulcers, serositis, proteinuria, anemia, leucopenia, thrombocytopenia, hemolytic anemia, anti-Sm, or anti-DNA in SLE/Ps+ and SLE/PsA+ patients (Tables 2 and 3).

\section{Discussion}

Psoriasis is an autoimmune chronic inflammatory skin disease which affects around $3 \%$ of the adult population in the United States [11]. Psoriatic arthritis, an inflammatory arthritis with a varied clinical presentation, may affect up to one third of Ps patients, occasionally preceeding the skin manifestations [6].

The coexistence of Ps and SLE is uncommon, while the association of SLE and PsA has rarely been described in the medical literature.

Our study found that the percentage of SLE patients affected with Psoriasis is $5.1 \%$ and that $87 \%$ of patients with concomitant SLE and Ps were affected with PsA indicating a higher prevalence of PsA, but not of Ps in SLE patients $(p<0.0001)$. This suggests that the patients with Ps are predisposed to developing PsA with concomitant presence of SLE.

Dermatologic manifestations of SLE such as malar rash, discoid lupus and photosensitivity occurred more frequently in patients with Ps, whereas the presence of APLA was less frequent in this group (Table 1). The association between APLA and Ps has been looked at before with results showing no increased prevalence of APLA in Ps patients [12].

To the best of our knowledge this is the largest series of patients with concomitant PsA and SLE. In this series of patients, we show an increased risk for developing arthritis in Ps patients with SLE when compared to Ps patients without SLE. Multiple reasons may be behind this association.

A common etiopathogenic factor for both diseases is IL17 a proinflammatory molecule that is associated with chronic inflammation in Ps and may also play a role in the pathogenesis of SLE. IL-17 production is known to be increased in SLE patients and it has an important role in the inflammation seen in SLE as it may be associated with T-cell mediated injury but also with autoantibody production. In animals, it has been shown that blockade of IL-17 decreases SLE manifestations [13,14].

In regards to PsA, it has been shown that IL-17+ CD4- T cells are present at increased levels in synovial fluid of PsA patients when compared to paired samples of peripheral blood in PsA and to synovial fluid from RA patients [15].

Ustekinumab is a human monoclonal antibody that binds to the shared p40 subunit of interleukin (IL)-12 and IL-23, blocking the signal of their receptors, and therefore blocking the Th1 and Th17 signaling pathways. It has been approved by the FDA for the treatment of PsA since September of 2013. Secukinumab and ixekizumab (anti-IL-17A monoclonal antibodies) are currently being tested in early randomized controlled trials with initial good results [16].

Studies have suggested that interferon-alpha (IFN- $\alpha$ ) may have a role in pathogenesis of Ps, and that Type I interferon pathways are upregulated in early lesions of psoriasis $[17,18]$. Recombinant human IFN- $\alpha$ has been used in the treatment of several cancers, but there have been several reports of it exacerbating or triggering Ps. There have also been case reports of pegylated IFN- $a-2 b$ inducing Ps. The role of elevated interferon-alpha has been well established in the pathogenesis of SLE by promoting feedback loops progressively disrupting peripheral immune tolerance and driving disease activity $[19,20]$.

Limitations of this study include the fact that it is a retrospective chart review from a single tertiary referral center and the absence of pathologic confirmation of the skin Ps diagnosis. No causative or chronographic developments could be induced from this study but does provide enough evidence for hypothesis development and further

Table 1. Prevalence of SLE diagnostic criteria in patients with (SLE/Ps + ) and without psoriasis (SLE/Ps + ) as well as in patients with (SLE/PsA + ) and without psoriatic arthritis (SLE/Ps + ) in a cohort of 445 SLE subjects. Following table shows features with statistically significant $p$ value.

\begin{tabular}{|c|c|c|c|c|c|c|c|c|c|c|}
\hline & \multicolumn{2}{|c|}{ SLE/Ps +} & \multirow{2}{*}{$\begin{array}{c}\text { SLE/Ps- } \\
\text { Present }\end{array}$} & \multirow[b]{2}{*}{ Absent } & \multirow[t]{2}{*}{$p$ value } & \multicolumn{2}{|c|}{ SLE/PsA+ } & \multicolumn{2}{|c|}{ SLE/PsA- } & \multirow[t]{2}{*}{$p$ value } \\
\hline & Present & Absent & & & & Present & Absent & Present & Absent & \\
\hline Malar rash & 11 & 12 & 118 & 304 & 0.0409 & 10 & 10 & 119 & 306 & 0.0341 \\
\hline Discoid rash & 4 & 19 & 21 & 401 & 0.0118 & 4 & 16 & 21 & 404 & 0.0043 \\
\hline Photosensitivity & 13 & 10 & 142 & 280 & 0.025 & 12 & 8 & 143 & 282 & 0.0156 \\
\hline Arthritis & 21 & 2 & 211 & 211 & 0.0001 & 20 & 0 & 213 & 212 & $<0.0001$ \\
\hline APL Antibodies & 1 & 22 & 108 & 314 & 0.021 & 1 & 19 & 108 & 317 & 0.038 \\
\hline
\end{tabular}


Table 2. Prevalence of SLE diagnostic criteria in patients with (SLE/Ps + ) and without psoriasis (SLE/Ps + ) in a cohort of 445 SLE subjects. Following table shows features which did not meet statistical significance.

\begin{tabular}{|l|c|c|c|c|c|}
\hline & \multicolumn{2}{|c|}{ SLE/Ps+ } & \multicolumn{2}{c|}{ SLE/Ps- } & p value \\
\hline & Present & Absent & Present & Absent & \\
\hline Serositis & 1 & 22 & 28 & 394 & 0.6652 \\
\hline Proteinuria & 1 & 22 & 42 & 380 & 0.3756 \\
\hline Neurological disorders & 3 & 20 & 67 & 355 & 0.7163 \\
\hline Anemia & 2 & 21 & 40 & 382 & 0.9005 \\
\hline Leukopenia & 2 & 21 & 23 & 399 & 0.5104 \\
\hline Thrombocytopenia & 1 & 22 & 28 & 394 & 0.6652 \\
\hline Hemolytic anemia & 1 & 22 & 4 & 418 & 0.1319 \\
\hline Anti-Sm antibody & 1 & 22 & 28 & 394 & 0.6652 \\
\hline Anti-ds-DNA antibody & 3 & 20 & 99 & 323 & 0.2471 \\
\hline
\end{tabular}

Table 3. Prevalence of SLE diagnostic criteria in patients with (SLE/PsA + ) and without psoriatic arthritis (SLE/PsA + ) in a cohort of 445 SLE subjects. Following table shows criteria which did not statistically correlate with diagnosis.

\begin{tabular}{|l|c|c|c|c|c|}
\hline \multicolumn{2}{|c|}{ SLE/PsA+ } & \multicolumn{2}{c|}{ SLE/PsA- } & p value \\
\hline & Present & Absent & Present & Absent & \\
\hline Serositis & 0 & 20 & 29 & 396 & 0.227 \\
\hline Proteinuria & 0 & 20 & 43 & 382 & 0.1345 \\
\hline Neurological disorders & 2 & 18 & 68 & 357 & 0.4714 \\
\hline Anemia & 1 & 19 & 41 & 384 & 0.4714 \\
\hline Leukopenia & 2 & 18 & 23 & 402 & 0.383 \\
\hline Thrombocytopenia & 1 & 19 & 28 & 397 & 0.7785 \\
\hline Hemolytic anemia & 0 & 20 & 5 & 425 & 0.5927 \\
\hline Anti-Sm antibody & 0 & 20 & 29 & 396 & 0.2027 \\
\hline Anti-ds-DNA antibody & 3 & 17 & 99 & 326 & 0.3768 \\
\hline
\end{tabular}

studies. Future prospective studies need to be developed in multiple centers to capture a wider range of disease characteristics.

In conclusion, we recognized an increased prevalence of PsA in patients with SLE. The coexistence has not been well described, as inflammatory arthritis could be part of PsA or SLE. This poses a diagnostic and therapeutic challenge to the treating clinician. Larger registry studies are needed to further delineate this prevalence and identify various factors which may help in both diagnosis and management of the patient. Delineating the pathogenesis further might help us develop better treatment options.

\section{References}

1. Danchenko N, Satia JA, Anthony MS (2006) Epidemiology of systemic lupus erythematosus: a comparison of worldwide disease burden. Lupus 15: 308-318. [Crossref]

2. Zalla MJ, Muller SA (1996) The coexistence of psoriasis with lupus erythematosus and other photosensitive disorders. Acta Derm Venereol Suppl (Stockh) 195: 1-15. [Crossref]

3. Wohl Y, Brenner S (2004) Cutaneous LE or psoriasis: a tricky differential diagnosis. Lupus 13: 72-74. [Crossref]
4. Nestle FO, Kaplan DH, Barker J (2009) Psoriasis. N Engl J Med 361: 496-509. [Crossref]

5. Toichi E, Torres G, McCormick TS, Chang T, Mascelli MA, et al. (2006) An antiIL-12p40 antibody down-regulates type 1 cytokines, chemokines, and IL-12/IL-23 in psoriasis. J Immunol 177: 4917-4926. [Crossref]

6. Mease PJ, Gladman DD, Papp KA, Khraishi MM, Thaci D, et al. (2013) Prevalence of rheumatologist-diagnosed psoriatic arthritis in patients with psoriasis in European/ North American dermatology clinics. J Am Acad Dermatol 69: 729-735. [Crossref]

7. Janjumratsang P, Phainupong D, Chanjanakijskul S, Roongphibulsopit P (2008) Positive direct immunofluorescence and autoantibody profiles in psoriasis patients. $J$ Dermatol 35: 508-513. [Crossref]

8. Avriel A, Zeller L, Flusser D, Abu Shakra M, Halevy S, et al. (2007) Coexistence of psoriatic arthritis and systemic lupus erythematosus. Isr Med Assoc J 9: 48-49. [Crossref]

9. Hochberg MC (1997) Updating the American College of Rheumatology revised criteria for the classification of systemic lupus erythematosus. Arthritis Rheum 40: 1725. [Crossref]

10. Taylor W, Gladman D, Helliwell P, Marchesoni A, Mease P, et al. (2006) Classification criteria for psoriatic arthritis: Development of new criteria from a large international study. Arthritis Rheum 54: 2665-2673. [Crossref]

11. Hamilton MP, Ntais D, Griffiths CE, Davies LM; Identification and Management of Psoriasis-Associated ComorbidiTy (IMPACT) Team (2015) Psoriasis treatment and management - a systematic review of full economic evaluations. Br J Dermatol 172: 574-583. [Crossref]

12. Cassano N, Buquicchio R, Ranieri V, Loconsole F, Vena GA (2008) Is there an association between antiphospholipid antibodies and psoriasis? J Biol Regul Homeost Agents 22: 207-210. [Crossref]

13. Nalbandian A1, Crispín JC, Tsokos GC (2009) Interleukin-17 and systemic lupus erythematosus: current concepts. Clin Exp Immunol 157: 209-215. [Crossref]

14. Hsu HC, Yang P, Wang J, Wu Q, Myers R, et al. (2008) Interleukin 17-producing T helper cells and interleukin 17 orchestrate autoreactive germinal center development in autoimmune BXD2 mice. Nat Immunol 9: 166-175. [Crossref]

15. Menon B, Gullick NJ, Walter GJ, Rajasekhar M, Garrood T, et al. (2014) Interleukin$17+\mathrm{CD} 8+\mathrm{T}$ cells are enriched in the joints of patients with psoriatic arthritis and correlate with disease activity and joint damage progression. Arthritis Rheumatol 66: 1272-1281. [Crossref]

16. McInnes IB, Mease PJ, Kirkham B, Kavanaugh A, Ritchlin CT, et al (2015) Articles: Secukinumab, a human anti-interleukin-17A monoclonal antibody, in patients with psoriatic arthritis (FUTURE 2): a randomised, double-blind, placebo-controlled, phase 3 trial. Lancet 386: 1137-1146. [Crossref]

17. Van Der Fits L, Van Der Wel LI, Laman JD, Prens EP, Verschuren MCM (2004) In Psoriasis Lesional Skin the Type I Interferon Signaling Pathway Is Activated, whereas Interferon-?? Sensitivity Is Unaltered. J Invest Dermatol 122: 51-60. [Crossref]

18. Funk J, Langeland T, Schrumpf E, Hanssen LE (1991) Psoriasis induced by interferonalpha. Br J Dermatol 125: 463-465. [Crossref]

19. Feng X, Wu H, Grossman JM, Hanvivadhanakul P, FitzGerald JD, et al. (2006) Association of increased interferon-inducible gene expression with disease activity and lupus nephritis in patients with systemic lupus erythematosus. Arthritis Rheum 54: 2951-2962. [Crossref]

20. Obermoser G, Pascual V (2010) The interferon-alpha signature of systemic lupus erythematosus. Lupus 19: 1012-1019. [Crossref]

Copyright: (C2016 Bonilla E. This is an open-access article distributed under the terms of the Creative Commons Attribution License, which permits unrestricted use, distribution, and reproduction in any medium, provided the original author and source are credited. 